\title{
EMITTANCE COUPLING IN HIGH INTENSITY BEAMS APPLIED TO THE SNS LINAC
}

\author{
I. Hofmann, G. Franchetti, O. Boine-Frankenheim, GSI, Darmstadt, Germany \\ J. Qiang, R. Ryne, LANL, Los Alamos, NM 87545, USA \\ D. Jeon and J. Wei, ORNL, Oak Ridge, TN 37831, USA
}

\begin{abstract}
Space charge can lead to emittance and/or energy exchange in non-equipartitioned high intensity linacs, which is interpreted here in terms of "coherent resonance". In this paper we first compare 2D and 3D simulations in constant focusing, and relate them to self-consistent resonant charts based on analytical theory. We then use these charts to interpret PARMILA simulation results for the SNS linac and find that the SNS design shows little sensitivity to such resonances for the nominal (longitudinal to transverse) emittance ratio; with doubled input longitidinal emittance we have identified modest emittance transfer by crossing of the fourth order 2:2 resonance stopband in the DTL and SRF.
\end{abstract}

\section{INTRODUCTION}

Emittance or energy exchange in space charge dominated beams is of interest to identify possible sources of beam quality degradation for high-power proton linear accelerators [1], which is studied here with reference to the Spallation Neutron Source [2]. In 3D simulation studies of bunched beams in constant focusing, and for fixed longitudinal to transverse emittance ratio, it was shown that nonequipartitioned beams are not subject to energy/emittance exchange in certain regions of parameter space, while in other regions - associated with internal resonances - exchange in the direction of equipartition may occur [3]. The mechanism is that collective oscillations of the space charge density induce nonlinear forces (similar to those of magnetic octupoles or sextupoles), which lead to coupling if resonant motion is involved. A self-consistent description of this mechanism of collectively driven resonances was studied in detail in Ref. [4] using a Vlasov perturbation approach to the anisotropic 2D Kapchinskij-Vladimirskij (KV) distribution. In a recent 2D simulation study for a limited range of parameters (emittance ratio 2; vicinity of the potentially most dangerous $2: 2$ resonance at tune ratio 1) it was shown by a comparison of KV-beams with waterbag (WB) distributions that the stability of WB beams is well described by the non-oscillatory eigenmodes of the $\mathrm{KV}$-theory, and that the corresponding analytical growth rates can be used to develop practically useful resonance charts [5]. For emittance ratio 2 such a chart has been recently applied to the CERN superconducting linac study to interpret the output of 3D IMPACT code simulations [6].

\section{2D/3D SIMULATION BASIS FOR RESONANCE CHARTS}

To check the relative importance of the different resonances suggested by the resonance charts - and existence of possible higher order resonances - we first compare the behavior of $2 \mathrm{D}$ and $3 \mathrm{D}$ waterbag simulations under constant focusing. Note that 3 parameters are needed to characterize the rms properties of anisotropic beams with two degrees of freedom, where $z$ and $x$ are used for the longitudinal rsp. transverse degree of freedom. The energy anisotropy $T$ describing deviation from equipartition can be written in the harmonic oscillator approximation as $T \equiv \epsilon_{z} k_{z} / \epsilon_{x} k_{x}$. Here we assume that in the $3 \mathrm{D}$ case the initial distribution in $y$ is identical to that in $x$, whereas the $2 \mathrm{D}$ model ignores this direction by assuming the beam has infinite extent in $y$. In both cases the input is a waterbag distribution rms matched to a constant focusing system with tune ratios $k_{z} / k_{x}$ varied over a sufficiently large range, while the rms emittance ratio $\epsilon_{z} / \epsilon_{x}$ is kept constant. For the transverse tune depression we assume $k_{x} / k_{0 x}=0.5$, which characterizes the situation in the SNS linac for the maximum current. The 2D simulations in $z$ and $x$ have been carried out with the MICROMAP code [8] extended to 2D particle-incell and using 50k simulation particles on a grid of $128 \times 128$ cells. Calculations have been performed on the GALAXY parallel cluster at Brookhaven National Laboratories with a special script to allow an automated scan over the tune ratio for given emittance ratio and fixed tune depression in $x$. Each scan consists of typically 100 different working points $k_{z} / k_{x}$ to resolve possible higher order resonances. For the 3D simulations we have used the IMPACT particlein-cell code as described in Ref. [3], which employs a fully 3D Poisson solver with open boundary conditions on a grid of $64 \times 64 \times 64$ cells with 2000k simulation particles. In Fig. 1 we show results for the relative change of emittances at the end of simulation for two different emittance ratios, where $\epsilon_{z} / \epsilon_{x}=1.2$ refers to the nominal case of the SNS linac, and each marker refers to one simulation. Simulations were extended over 100 betatron periods, which was found sufficient to reach saturation in most cases - for the fourth order mode resonance the saturation occurs as fast as over five (zero-space charge) betatron periods at the peak exchange of Fig. 1, bottom. We introduce the nomenclature of "n:m resonance" to describe an internal (difference) resonance condition $m k_{z}-n k_{x}+\Delta \omega=0$ [4]; here $\Delta \omega$ is a coherent tune shift determining the width of the stopband, $n+m$ the resonance order, and $n / m$ the tune ra- 


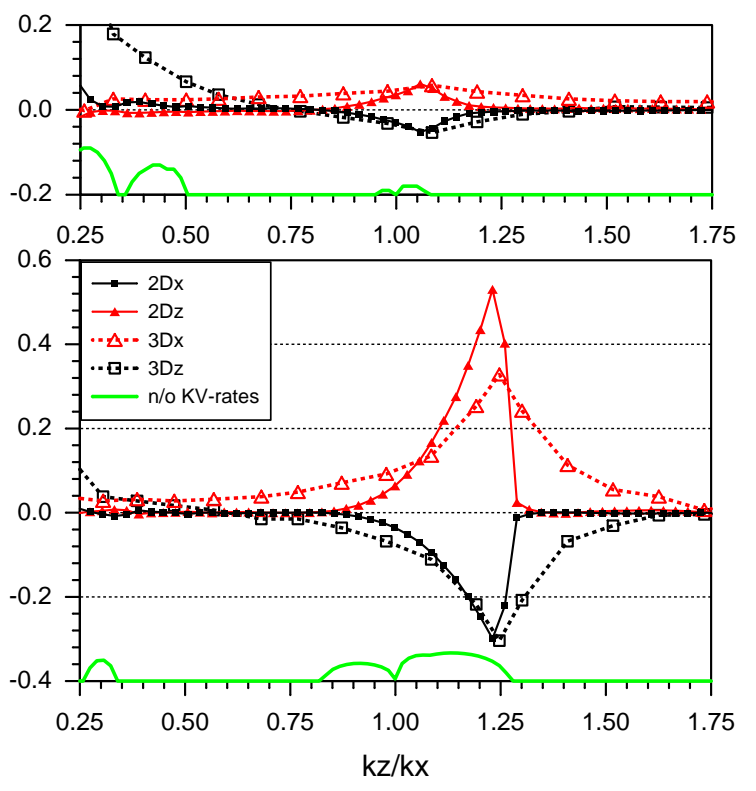

Figure 1: Emittance growth factors (units $\Delta \epsilon / \epsilon$ ) for constant focusing initial WB simulation of 2D MICROMAP tune scans and 3D IMPACT cases, compared with maximum growth rates (shown on bottom in scaled units) for $k_{x} / k_{0 x}=0.5$ and $\epsilon_{z} / \epsilon_{x}=1.2$ (top) and 2 (bottom).

tio to which the stopband converges for vanishing space charge. The 2D and 3D simulations show the pronounced $2: 2$ resonance for the emittance ratio 2 , related in position and width to the stopbands of the non-oscillatory modes of the KV-theory up to fourth order (as shown on the bottom lines of Fig. refscans). A not unexpected result is that emittance transfer in the resonance bands vanishes if the beam is equipartitioned there, since there is no free energy available for transfer. Exchange in the 2:2 stopband shrinks to about $5 \%$ for the emittance ratio 1.2 due to the vicinity of equipartitioning. The theoretically predicted stopband near tune ratio $1 / 2$ (1:2 third order mode) and 1/3 (1:3 fourth order mode) are also reflected by the simulations, but their effect on emittance transfer is found to be much less. The absence of stopbands for higher than fourth order modes is evident from the more refined tune scans in 2D. The comparison confirms that equipartition in 3D implies energy balance between the longitudinal degree of freedom with each one of the transverse degrees of freedom - and not the sum of transverse energies. It should be noted that the energy transferred from longitudinal to transverse is shared by $x$ and $y$, which may explain why the maximum transverse emittance increase in $3 \mathrm{D}$ is found less than in $2 \mathrm{D}$, while the decrease in $z$ is nearly identical. A striking (yet to be explained) difference is the symmetry of the $2: 2$ stopband in the 3D simulations for emittance ratio 2 that is not reflected by the $2 \mathrm{D}$ and analytical theory. The transfer occurs in the core with almost no halo: the ratio of maximum size to rms size grows from 2.45 for the initial 2D WB by at most $20 \%$ in the most significant stopband of Fig. refscans; in $3 \mathrm{D}$ the corresponding increase is $28 \%$ (typical halo radii for the 2:1 mismatch driven parametric halo are 4-5 times the rms radius [7]).

\section{APPLICATION TO THE SNS-LINAC}

Our theoretical study suggests that the issue of coupling resonances is not influenced by acceleration as such, but resonance conditions and growth rates only depend on changes of the emittance and tune ratios, and of tune depression; tune ratios are, of course, subject to variations caused by the varying rf phase and amplitude as well as $\beta \gamma$ and the accelerating structure. The SNS linac is a highcurrent linac designed for $2 \mathrm{MW}$ of $1 \mathrm{GeV} \mathrm{H}^{-}$, with a DTL and a CCL followed by the superconducting SRF [2]. The high power requires careful control of emittance blow-up and beam halo, which is collimated in the HEBT line both in the transverse directions and in momentum space. The primary concern is transverse beam blow-up, which results in ring injection foil miss, foil traversal and scattering increase, and radio-activation increase. Longitudinally, the momentum spread needs to be controlled within the linear correction range of the HEBT energy corrector to facilitate momentum painting. To explore the sensitivity of the SNS design to space charge resonances we assume an initially matched waterbag distribution with the "nominal" emittances taken from the RFQ output (initial $\epsilon_{z} / \epsilon_{x}=1.05$ ), compared with a doubled input longitudinal emittance. Our simulations have been performed with PARMILA using the $\mathrm{r}-\mathrm{z}$ space charge routine SCHEFF.

In Fig. 2 we show the theoretical chart for emittance ratio 1.2 - noting that the simulation initial emittance ratio is 1.05 , but it approaches an average of $1.2-$, and plot the tune trajectory up to the end of the DTL $(87 \mathrm{MeV})$, which extends over 8 zero space charge betatron and 37 lattice periods. The fourth order resonance stopband near tune ratio

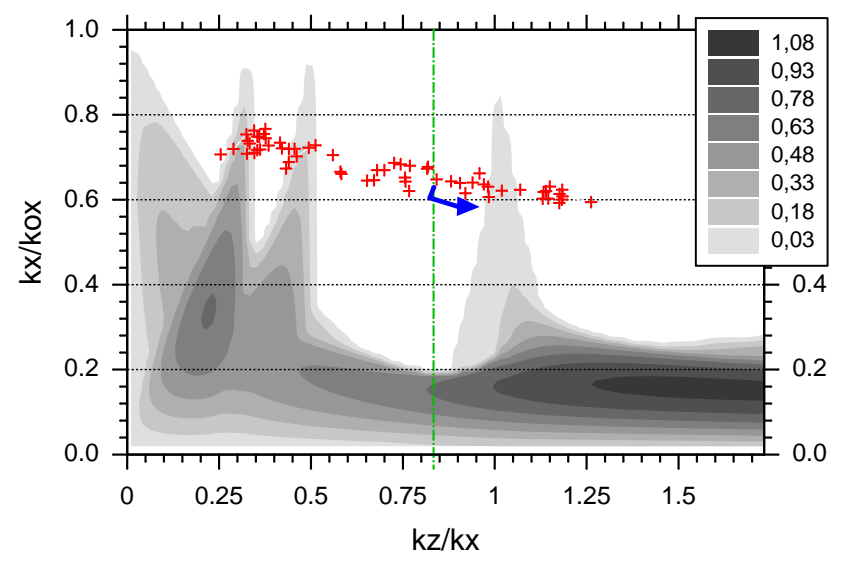

Figure 2: Theoretical resonance chart for $\epsilon_{z} / \epsilon_{x}=1.2$ with PARMILA tune trajectory through DTL for nominal SNS input emittances (at arrow). Grey scales indicate theoretical growth rates in units of inverse zero-current betatron periods per e-folding (vertical line marks equipartition). 
1 is very weak as the beam is almost equipartitioned there, with energy anisotropy $\approx 1.2$ (for an initial emittance ratio of 1 this resonance disappears, whereas all others remain similar). Consistently, crossing of it in the first tank of the DTL has no effect as is shown in Fig. 3. From Fig. 2 it is seen that - due to reduced longitudinal focusing - the 1:2 third order resonance (near tune ratio 1/2) is crossed next in the DTL. In accordance with Fig. 1 its saturation emittance transfer is small, in addition it would take many betatron periods as it needs to grow from initial noise. PARMILA would actually not properly resolve a third order mode, which violates the $r-z$ symmetry. The potentially more dangerous $1: 3$ fourth order resonance at tune ratio $1 / 3$ is hardly reached at the end of the DTL, and no effect is seen in the simulation of Fig. 3. The chart for emittance ratio

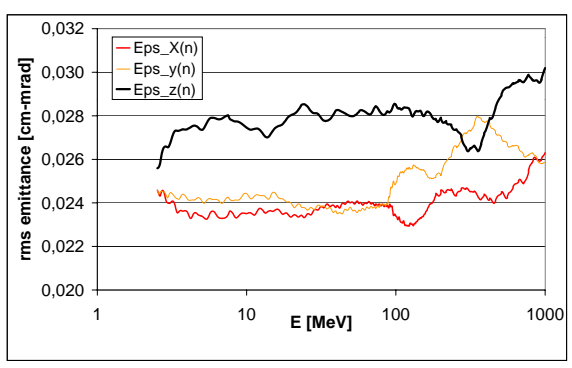

Figure 3: PARMILA result to full energy showing normalized emittances and using nominal input emittances.

2 is shown in Fig. 4; also the PARMILA tune trajectory within the DTL for a simulation with the same transverse input emittance as before, but the longitudinal one raised to $\epsilon_{z} / \epsilon_{x}=2.07$ ), which might result from errors/mismatch in the MEBT transfer line. Rematching to the linac lattice is performed in the first cell of the DTL. The 2:2 stopband is now significant due to the energy anisotropy of $\approx 2$. Crossing of it in the first DTL tank takes place in about 1-2 betatron periods, which is only a fraction of the theoretical e-folding time for exchange (about 5 betatron periods). In fact, Fig. 5 shows a visible exchange of emittances in the first tank of the DTL, which is only about $1 / 3$ rd of the possible maximum exchange according to Fig. 1. At higher energy in the SRF the tune ratio returns to the $2: 2$ stopband, and we observe further emittance exchange between 200 and $300 \mathrm{MeV}$ to almost the maximum expected from Fig. 1. As required by SCHEFF, this case preserves much better the symmetry in $x$ and $y$ than the case of Fig. 3 .

We note that these results need to be cross-checked with a fully $3 \mathrm{D}$ space charge calculation in the near future, although we assume that the case of the predominantly fourth order resonance identified here is sufficiently well reproduced by the r-z Poisson solver of PARMILA. 3D mismatch and error studies as well as halo formation will be the subject of further investigation; exploratory PARMILA simulations have indicated that a longitudinal mismatch factor of 1.3 has almost no effect on the transverse emit- tance for the nominal emittance ratio; for the doubled emittance ratio both effects work in the same direction and lead to an overall transverse rms emittance growth of $45 \%$ (at $1 \mathrm{GeV}$ ) compared with the nominal case and no mismatch.

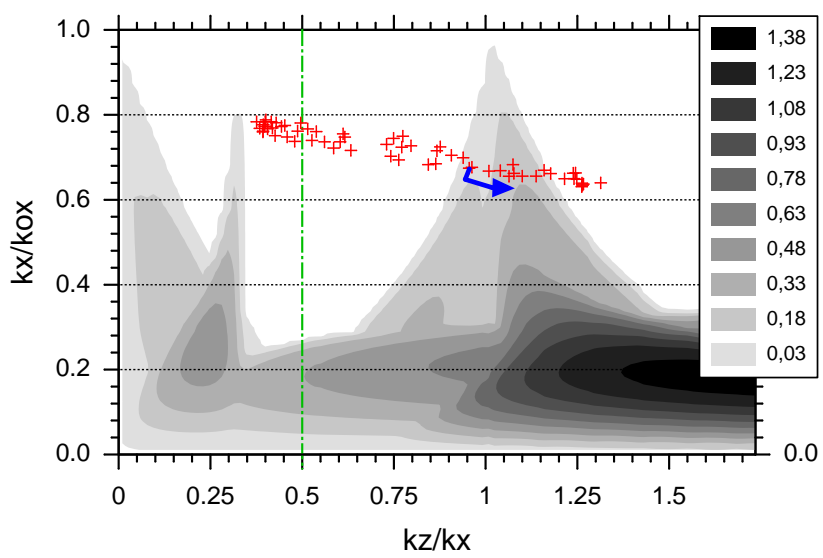

Figure 4: Same as Fig. 2 for $\epsilon_{z} / \epsilon_{x}=2$, and trajectory for doubled initial longitudinal emittance in PARMILA.

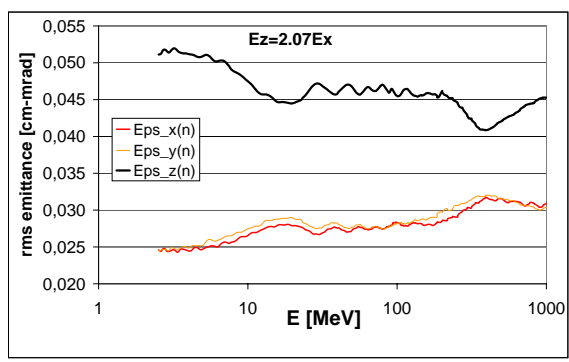

Figure 5: PARMILA result with doubled longitudinal input emittance.

Acknowledgment The hospitality and support of the SNS Project Office/ORNL, and of Brookhaven National Laboratories, are gratefully recognized by two of the authors (I.H. and G.F.).

\section{REFERENCES}

[1] R.A. Jameson, in Proceedings of the Third Workshop on Advanced Accelerator Concepts, Port Jefferson, 1992 (AIP Conf. Proc. 279), p. 969

[2] J. Stovall et al., LINAC 2000, p.605

[3] I. Hofmann, J. Qiang and R. Ryne, Phys. Rev. Lett. 86, 2313 (2001).

[4] I. Hofmann, Phys. Rev. E 57, 4713 (1998).

[5] I. Hofmann and O. Boine-Frankenheim, accepted for publication in Phys. Rev. Lett. (2001).

[6] F. Gerigk and I. Hofmann, these Proceedings

[7] T.P. Wangler, Phys. Rev. ST-AB 1, 084201 (2000).

[8] G. Franchetti, I. Hofmann, and G. Turchetti AIP Conf. Proc. 448, 233 (1998); ed. A.U.Luccio and W.T. Weng. 